\title{
Deformed Heisenberg algebra and minimal length
}

\author{
T. Masłowski ${ }^{1 *}$, A. Nowicki ${ }^{1 \dagger}$, V. M. Tkachuk ${ }^{2 \ddagger}$ \\ ${ }^{1}$ Institute of Physics, University of Zielona Góra \\ Prof. Z. Szafrana 4a, 65-516 Zielona Góra, Poland \\ ${ }^{2}$ Department of Theoretical Physics, \\ Ivan Franko National University of Lviv, \\ 12 Drahomanov St., Lviv, UA-79005, Ukraine
}

July 30, 2018

\begin{abstract}
A one-dimensional deformed Heisenberg algebra $[X, P]=i f(P)$ is studied. We answer the question: For what function of deformation $f(P)$ there exists a nonzero minimal uncertainty in position (minimal length). We also find an explicit expression for the minimal length in the case of arbitrary function of deformation.
\end{abstract}

\section{Introduction}

Recently much attention has been devoted to the study of deformed Heisenberg algebras of different kinds. In this paper we focus on deformed algebras with minimal length. History of this subject is very long. Snyder's paper [1] was the first publication on this subject. In that paper the Lorentzcovariant deformed Heisenberg algebra leading to quantized space-time was

\footnotetext{
*T.Maslowski@proton.if.uz.zgora.pl

$\dagger$ †.Nowicki@if.uz.zgora.pl

†tkachuk@ktf.franko.lviv.ua
} 
proposed. For a long time there were only a few paper on this subject. The interest to deformed algebras was renewed after investigations in string theory and quantum gravity which suggest the existence of a nonzero minimal uncertainty in position following from the generalized uncertainty principle (GUP). In [2, 3] it was shown that GUP and nonzero minimal uncertainty in position can be obtained from a modified Heisenberg algebra, where in the right hand side of it a term proportional to squared momentum is added. Subsequently there were published many papers where different quantum system in space with deformed Heisenberg algebra was studied. They are one-dimensional harmonic oscillator with minimal uncertainty in position [2] and also with minimal uncertainty in position and momentum [4, 5], Ddimensional isotropic harmonic oscillator [6, 7], three-dimensional Dirac oscillator [8] and one-dimensional Coulomb problem [9], (1+1)-dimensional Dirac oscillator with Lorentz-covariant deformed algebra [10], three-dimensional Coulomb problem with deformed Heisenberg algebra in the frame of perturbation theory [11, 12, 13, 14, 15], singular inverse square potential with a minimal length [16, 17], ultra-cold neutrons in gravitational field with minimal length [18, 19, 20], composite system in deformed space with minimal length [21, 22].

In this paper we study a general deformation of one-dimensional Heisenberg algebra, when the right hand side of it is some function of momentum. As we know, up to now there has been no answer to the question about the existence of a minimal length in this general case. The aim of this paper is to fill this gap.

\section{Minimal length}

We consider a modified one-dimensional Heisenberg algebra generated by position $\mathbf{X}$ and momentum $\mathbf{P}$ hermitian operators satisfying

$$
[\mathbf{X}, \mathbf{P}]=i f(\mathbf{P})
$$

where $f$ is called function of deformation and we assume that it is strictly positive $(f>0)$, even function (cf. [2]).

In momentum representation both operators acting on a square integrable functions $\phi(p) \in \mathcal{L}^{2}(-a, a ; f),(a \leq \infty)$

$$
\mathbf{P} \phi(p)=p \phi(p)
$$




$$
\mathbf{X} \phi(p)=i f(p) \frac{d}{d p} \phi(p)
$$

where the norm of $\phi$ is given by

$$
\|\phi\|^{2}=\int_{-a}^{a} \frac{d p}{f(p)}|\phi(p)|^{2} .
$$

The hermiticity of $\mathbf{X}$ demands $\phi(-a)=\phi(a)=0$.

The aim of this paper is to answer the question: for what function of deformation $f(p)$ there exists a nonzero minimal uncertainty in position $\Delta_{\phi}(\mathbf{X}) \geq \Delta(\mathbf{X})_{\min }$. Nonzero minimal uncertainty in position $\Delta(\mathbf{X})_{\min }=l_{0}$ is called nonzero minimal length.

Further we use the following definitions of the mean value $\langle\mathbf{A}\rangle_{\phi}$ and dispersion $\Delta_{\phi}(\mathbf{A})$ of some operator $\mathbf{A}$ in the state $\phi \in \mathcal{L}^{2}(-a, a ; f)$

$$
\begin{aligned}
\langle\mathbf{A}\rangle_{\phi} & =\int_{-a}^{a} \frac{d p}{f(p)} \phi^{*}(p) \mathbf{A} \phi(p) \\
\Delta_{\phi}^{2}(\mathbf{A}) & =\left\langle\mathbf{A}^{2}\right\rangle_{\phi}-\langle\mathbf{A}\rangle_{\phi}^{2}=\int_{-a}^{a} \frac{d p}{f(p)} \phi^{*}(p)\left(\mathbf{A}-\langle\mathbf{A}\rangle_{\phi}\right)^{2} \phi(p),
\end{aligned}
$$

for normed states $\|\phi\|^{2}=\langle\mathbf{I}\rangle_{\phi}=1$.

Let us recall two well known facts which follow from the Heisenberg uncertainty relation

$$
\Delta_{\phi}^{2}(\mathbf{X}) \Delta_{\phi}^{2}(\mathbf{P}) \geq \frac{1}{4}\langle f(\mathbf{P})\rangle_{\phi}^{2}
$$

The first one is that for the non-deformed case when $f(p)=1$ the minimal length is zero. The second one states that in the case of function of deformation $f(p)=1+\beta p^{2}$ the minimal length is nonzero and reads [2, 3]

$$
l_{0}=\Delta(\mathbf{X})_{\min }=\sqrt{\beta} .
$$

For these two cases the momentum $p$ is given on the full line $-\infty<p<\infty$.

In the case of some other functions of deformation it is also possible to get minimal length using Heisenberg uncertainty relation. But in general case of arbitrary $f(p)$ it is difficult to find minimal length using (7) and to give answer about the existence of minimal length.

The idea of this paper is to relate the deformed algebra characterized by $f(P)$ to one of these two algebras, namely, either to the non-deformed 
$(f(P)=1)$ one or to the deformed one characterized by $f(P)=1+\beta P^{2}$. We find that the minimal length in the first case is zero and in the second is nonzero. Moreover we also find the value of the minimal length.

One can also consider nonlinearly transformed momentum operator $\mathbf{Q}=$ $h(\mathbf{P})$, where a function $q=h(p)$ is continuous, strictly increasing on interval $[-a, a]$ and operator $\mathbf{X}$ is the same for both algebras. Under this mapping we obtain a new deformed algebra related to (1) and satisfying the relation

$$
[\mathbf{X}, \mathbf{Q}]=i g(\mathbf{Q}), \quad g(q)=f(p) \frac{d q}{d p}
$$

and we assume that the function of deformation $g$ similarly as $f$ is a positive even function 1 .

Using function $q=h(p)$ we can change variable $\tilde{\phi}(h(p))=\phi(p)$ and we get realization of $\mathbf{Q}, \mathbf{X}$ in the space of square integrable functions $\tilde{\phi}(q) \in$ $\mathcal{L}^{2}(-b, b ; g)$

$$
\mathbf{Q} \tilde{\phi}(q)=q \tilde{\phi}(q), \quad \mathbf{X} \tilde{\phi}(q)=i g(q) \frac{d \tilde{\phi}}{d q}
$$

and the norms of state in both spaces are equal

$$
\|\phi\|^{2}=\int_{-a}^{a} \frac{d p}{f(p)}|\phi(p)|^{2}=\int_{-b}^{b} \frac{d q}{g(q)}|\tilde{\phi}(q)|^{2}=\|\tilde{\phi}\|^{2},
$$

which follows from (91). The same holds for dispersions

$$
\Delta_{\phi}^{2}(\mathbf{A})=\Delta_{\tilde{\phi}}^{2}(\mathbf{A})
$$

From the second equation in (9) we find relation between $p$ and $q$

$$
\int_{0}^{p} \frac{d p^{\prime}}{f\left(p^{\prime}\right)}=\int_{0}^{q} \frac{d q^{\prime}}{g\left(q^{\prime}\right)}
$$

which implicitly defines the transformation $q=h(p)$. In this case function $h(p)$ maps the domain $-a \leq p \leq a$ onto $-b=h(-a) \leq q \leq h(a)=b$. When such mapping is possible the minimal length will be the same for two algebras (1) and (9).

\footnotetext{
${ }^{1}$ This assumption means that $h(p)$ is an odd function.
} 
From (13) it follows that

$$
\int_{0}^{a} \frac{d p}{f(p)}=\int_{0}^{b} \frac{d q}{g(q)}
$$

which is equivalent that a mapping $q=h(p)$ from the domain $-a \leq p \leq a$ to the domain $-b \leq q \leq b$ is possible.

We consider two cases. The first case

$$
\int_{0}^{a} \frac{d p}{f(p)}=\infty
$$

In order to fulfill this condition we can put $g=1$ with $q$ given on the full line

$$
\int_{0}^{a} \frac{d p}{f(p)}=\int_{0}^{\infty} d q=\infty .
$$

So, in this case algebra (11) is mapped to non-deformed one $(g=1)$ on the full line and therefore the minimal lenght is zero.

In the second case

$$
\int_{0}^{a} \frac{d p}{f(p)}=\text { const }<\infty
$$

Now in order to fulfil (17) we can choose $g(q)=1+\beta q^{2}$ and $b=h(a)=\infty$. Then $\beta$ can be found from the equation

$$
\int_{0}^{a} \frac{d p}{f(p)}=\int_{0}^{\infty} \frac{d q}{1+\beta q^{2}}=\frac{\pi}{2 \sqrt{\beta}} .
$$

In this case algebra (1) is mapped to the deformed algebra proposed by Kempf [2, 3] and according to (8) the minimal length is

$$
l_{0}=\frac{\pi}{2}\left(\int_{0}^{a} \frac{d p}{f(p)}\right)^{-1} .
$$

Let us consider more explicitly a few examples.

Example 1.

$$
f(p)=e^{\alpha p^{2}}
$$

where $-\infty<p<\infty$. This function of deformation in the case $\alpha>0$ was recently considered in [23]. 
For $\alpha=\lambda^{2}>0$ using (19) we find that the minimal length is

$$
l_{0}=\lambda \sqrt{\pi} .
$$

In the case $\alpha \leq 0$ the minimal length is zero.

For the case of $\alpha=\lambda^{2}$ it is also possible to find the minimal length in another way using the fact that in this case the function of deformation as function of $p^{2}$ is convex. As a result the Heisenberg uncertainty relation (7) reads 23

$$
\Delta_{\phi}^{2}(\mathbf{X}) \Delta_{\phi}^{2}(\mathbf{P}) \geq \frac{1}{4}\left\langle e^{\lambda^{2} \mathbf{P}^{2}}\right\rangle_{\phi}^{2} \geq \frac{1}{4} e^{2 \lambda^{2}\left\langle\mathbf{P}^{2}\right\rangle_{\phi}},
$$

and one can find that

$$
l_{0}=\lambda \sqrt{\frac{e}{2}} .
$$

As we see our method gives a better result for minimal length (21) in comparison with (23).

Example 2.

$$
f(p)=\left(1+\lambda^{2} p^{2}\right)^{\alpha}
$$

where $-\infty<p<\infty$.

For $\alpha \leq 1 / 2$ the minimal length is zero and in the case of $\alpha>1 / 2$ the minimal length reads

$$
l_{0}=\lambda \frac{\sqrt{\pi} \Gamma(\alpha)}{\Gamma(\alpha-1 / 2)} .
$$

It is worth to note that in the case of $\alpha>1$ the function of deformation as function of $p^{2}$ is convex and using this fact it is possible to get some result for minimal length similarly as in the first example. For other $\alpha$ the function of deformation is not convex, nevertheless our method gives a possibility to obtain the result for the minimal length.

Example 3. To make Example 2 complete let us consider

$$
f(p)=\left(1-\lambda^{2} p^{2}\right)^{\alpha},
$$

where $-1 / \lambda \leq p \leq 1 / \lambda$.

We find that for $\alpha \geq 1$ the minimal length is zero and for $\alpha<1$ we obtain

$$
l_{0}=\lambda \frac{\sqrt{\pi} \Gamma(3 / 2-\alpha)}{\Gamma(1-\alpha)} .
$$




\section{Conclusion}

In this paper we have studied deformed algebras (11) with a symmetric function of deformation and answered the following question: For what function of deformation $f(p)$ the minimal length is nonzero? Answer to this question is given by equation (19) which presents the minimal length in the case of an arbitrary function of deformation and is the main result of the paper. When $\int_{0}^{a} \frac{d p}{f(p)}$ is finite the minimal length is nonzero and when this integral diverges the minimal length is zero. Using (19) we can calculate an explicit expression for the minimal length for different functions of deformation which is demonstrated in this paper by several examples.

\section{Acknowledgment}

VMT thanks for warm hospitality the University of Zielona Góra where this paper was done.

\section{References}

[1] H. S. Snyder, Phys. Rev. 71, 38 (1947).

[2] A. Kempf, G. Mangano, R. B. Mann, Phys. Rev. D 52, 1108 (1995).

[3] A. Kempf, Phys. Rev. D 54, 5174 (1996).

[4] C. Quesne and V. M. Tkachuk, J. Phys. A 36, 10373 (2003).

[5] C. Quesne and V. M. Tkachuk, J. Phys. A 37, 10095 (2004).

[6] L. N. Chang, D. Minic, N. Okamura and T. Takeuchi, Phys. Rev. D 65, 125027 (2002).

[7] I. Dadić, L. Jonke and S. Meljanac, Phys. Rev. D 67, 087701 (2003).

[8] C. Quesne and V. M. Tkachuk, J. Phys. A 38, 1747 (2005).

[9] T. V. Fityo, I. O. Vakarchuk and V. M. Tkachuk, J. Phys. A 39, 2143 (2006).

[10] C. Quesne and V. M. Tkachuk, J. Phys. A 39, 10909 (2006). 
[11] F. Brau, J. Phys. A 32, 7691 (1999).

[12] S. Benczik, L. N. Chang, D. Minic and T. Takeuchi, Phys. Rev. A 72, 012104 (2005).

[13] M. M. Stetsko and V. M. Tkachuk, Phys. Rev. A 74, 012101 (2006).

[14] M. M. Stetsko, Phys. Rev. A 74, 062105 (2006).

[15] M. M. Stetsko and V. M. Tkachuk, Phys. Lett. A 372, 5126 (2008).

[16] Djamil Bouaziz, Michel Bawin, Phys.Rev.A 76, 032112 (2007).

[17] Djamil Bouaziz, Michel Bawin, Phys.Rev.A 78, 032110 (2008).

[18] F. Brau, F. Buisseret, Phys.Rev.D 74, 036002 (2006).

[19] Kourosh Nozari, Pouria Pedram, EPL 92, 50013 (2010).

[20] Pouria Pedram, Kourosh Nozari, S. H. Taheri, JHEP 1103:093, (2011).

[21] C. Quesne, V.M. Tkachuk, Phys. Rev. A 81, 012106 (2010).

[22] F. Buisseret, Phys.Rev. A 82, 062102 (2010).

[23] Glauber Dorsch, Jose Alexandre Nogueira, Minimal Length in Quantum Mechanics via Modified Heisenberg Algebra, arXiv:1106.2737 